\title{
In vitro study of the aortic interleaflet triangle reshaping
}

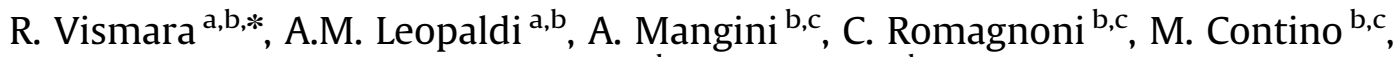 \\ C. Antona ${ }^{\mathrm{b}, \mathrm{c}}$, G.B. Fiore ${ }^{\mathrm{a}, \mathrm{b}}$ \\ a Department of Electronic, Information and Bioengineering, Politecnico di Milano, via Golgi 39, Milan 20133, Italy \\ ${ }^{\mathrm{b}}$ ForCardioLab, Fondazione per la Ricerca in Cardiochirurgia ONLUS, Milan, Italy \\ ${ }^{c}$ Cardiovascular Surgery Department, L. Sacco Hospital, University of Milan, Milan, Italy
}

\begin{abstract}
Aortic interleaflet triangle reshaping (AITR) is a surgical approach to aortic valve incontinence that involves placing three stitches at half of the interleaflet triangles height. In this work, the relationship between the actual stitch height and valve functioning, and the safety margin that the surgeon can rely on in applying the stitches were systematically investigated in vitro. AITR surgery was applied to six swine aortic roots placing the stitches empirically at $50 \%, 60 \%$ and $75 \%$ of the triangle heights. Then the actual stitch heights were measured and the hydrodynamic performances were evaluated with a pulsatile hydrodynamic mock loop. Actual stitch heights were $45 \pm 2 \%, 61 \pm 4 \%$ and $79 \pm 6 \%$. As compared to untreated conditions, the $50 \%$ configuration induced a significant variation in the effective orifice area. With stitches placed at $60 \%$, the mean systolic pressure drop increased significantly with respect to the untreated case, but no significant changes were recorded with respect to the 50\% configuration. At 75\%, all the hydrodynamic parameters of systolic valve functioning worsened significantly. Summarizing, the AITR technique, when performed in a conservative manner did not induce significant alterations in the hydrodynamics of the aortic root in vitro, while more aggressive configurations did. The absence of a statistically significant difference between the 50\% and 60\%configurations suggests that there is a reasonably limited risk of inducing valve stenosis in the post-op scenario due to stitch misplacement.
\end{abstract}

\section{Introduction}

The reparative approach to aortic valve (AV) surgery is feasible in case of AV regurgitation and has gained increasing interest in the last two decades (Cheng et al., 2007; Hopkins, 2003; Yacoub et al., 1999). Aortic regurgitation amounts for about $13 \%$ of total native valve diseases; the main etiology is degenerative, followed by congenital diseases and by rheumatic, endocarditis and inflammatory causes. The population mean age is $58 \pm 16$ years old (Vahanian et al., 2007). Resorting to valve repair has the benefit of sparing the native tissue, virtually bringing the repaired valve to function as a healthy one, avoiding any complications induced by the use of valve prostheses. Restoring the structure and function of the AV, which involves a complex interplay among the three leaflets and the other valvular substructures, has been challenged by the cardiac surgeons community in recent years, whereas applying reparative surgery techniques to the mitral valve is a consolidated practice since the seventies (Langer et al., 2004).

Keywords:

Interleaflet triangle reshaping

Aortic valve

Reparative surgery

In vitro tests

Corresponding author. Tel.: +3922399 4142; fax: +39223993360.

E-mail address: riccardo.vismara@polimi.it (R. Vismara).
Aortic interleaflet triangle reshaping (AITR) is a surgical reparative procedure applied in case of AV regurgitation secondary to sinotubular junction (STJ) dilatation or aortoventricular junction (AVJ) dilatation. At the Cardio Thoracic Unit of the Sacco Hospital, as well as at other surgical units focused on AV repair (Boodhwani et al., 2009a, 2009b; Mve Mvondo et al., 2013), AITR is intended as an ancillary surgical technique. Specifically, AITR is applied to valves which have already undergone a primary surgical repair, whose outcome is intended to be a continent $\mathrm{AV}$, with a correct functional anatomy. In this scenario, the aim of AITR is to stabilize valve continence in the mid- to long-term period, by preventing further dilatation of the STJ and/or AVJ and providing a functional leaflet coaptation reserve (Cabrol et al., 1966; El Khoury et al., 2005).

The AITR procedure involves placing a U suture, reinforced with e-PTFE pledgets, in correspondence of each interleaflet triangle (IT). In the anatomy of the IT (Fig. 1), the lateral sides are represented by the insertions of the aortic cusps, which follow diverging arcs proceeding centrally towards the apex of the heart. This feature makes the height of the stitches critical for the outcome of the AITR procedure (Fraser and Cosgrove, 1994). In particular, if the stitches are placed too centrally, aortic stenosis may be induced in the immediate post-operative scenario. 


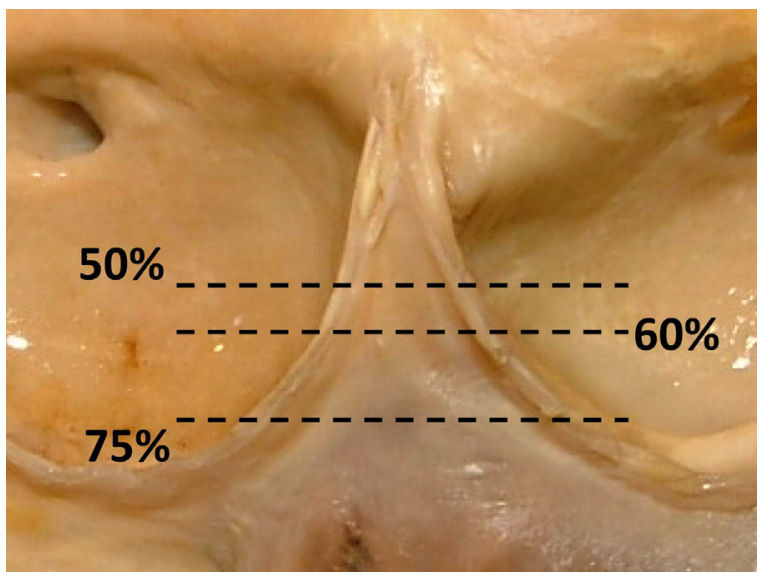

Fig. 1. Photography of the interleaflet triangles (IT). Dashed lines indicate the three heights at which stitches were placed. These heights were identified by the surgeons on the basis of visual inspection of the ITs.

The standard approach that is currently adopted at the Sacco Hospital (Mangini et al., 2011) consists in applying the stitches approximately at half of the interleaflet triangle height (ITH)(Fig. 1). This choice is intended to stabilize the AV without excessive narrowing of the ITs, thus avoiding clinically relevant stenosis, as confirmed by the available clinical and in vitro data (De Kerchove et al., 2012; Mangini et al., 2011).

Nevertheless, the execution of this procedure in the operating room presents two critical aspects that might affect the function of the repaired valve. First, intraoperatively the valve is in unloaded conditions. The surgeon has to figure out what will be the corrected valve morphology in its loaded conditions on the sole basis of his/ her experience, and place the stitches accordingly. Second, the identification of the height at which the stitches should be placed is based on a visual inspection of the valve and, being the surgical act a manual procedure, the actual height at which the stitch is materially applied is also subject to an error.

In the present study, using a consolidated in vitro approach (De Kerchove et al., 2012; Vismara et al., in press, 2011, 2010), we systematically analyzed the effects induced on the AV hemody-namic function by AITR procedures performed at three different heights. Our study had a twofold goal: (i) to quantify the relation-ship between the height of the stitches and the alteration of the indexes that characterize valve functioning, and (ii) to estimate the safety margin that the surgeon can rely on in the empirical choice of the proper stitch height for the surgery.

\section{Materials and methods}

\subsection{Mock loop layout}

The mock loop (Fig. 2) (Lanzarone et al., 2009; Vismara et al., 2010) consisted of (i) a PC-driven pulsatile volumetric pump able to replicate left ventricular flow waveforms, (ii) a sample test section designed to house the whole AV, and (iii) an adjustable hydraulic afterload mimicking the hydraulic input impedance of the systemic circulation. The sample housing section was adaptable to aortic roots of different sizes, and its design allowed surgeons to work in the laboratory simulating the conditions of the operating room (OR). The mock loop was equipped with a transit-time flow meter (HT110R, Transonic System Inc., Ithaca, NY) placed downstream of the ARFU sample, and three pressure transducers (PC140 series, Honeywell Inc., Morristown, NJ), two of which placed immediately upstream from and downstream of the sample ( $P_{\text {ven }}$ and $P_{\text {ao }}$ respectively in Fig. 2$)$, and one placed at the inlet section of the hydraulic afterload $\left(P_{\text {art }}\right)$. Data were acquired at a $200 \mathrm{~Hz}$ sampling rate via an A/D board (USB 6210, National Instruments, Austin, TX).

\subsection{In vitro AITR surgery}

The AITR procedures were performed by an experienced cardiac surgeon using a non-absorbable, braided 2-0 stitch reinforced with e-PTFE pledget. The surgery

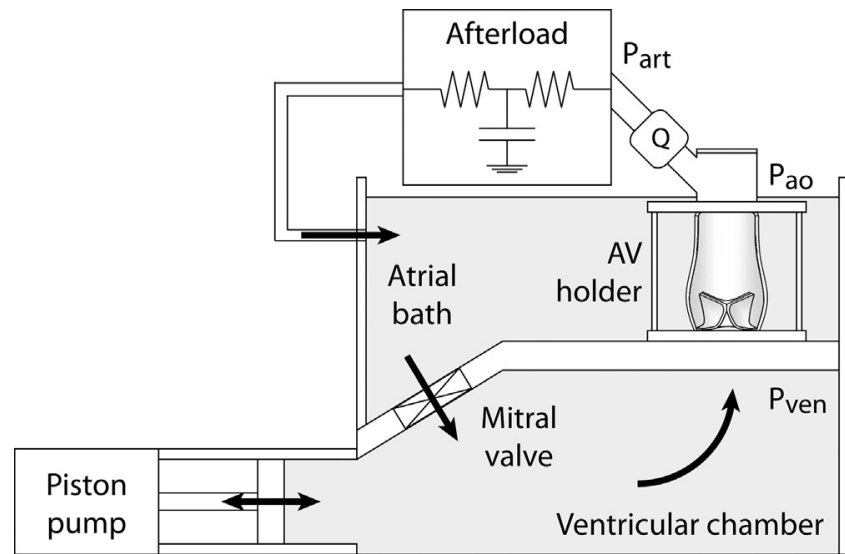

Fig. 2. Schematic of the in vitro mock loop. $P_{\text {ven }}$ : simulated ventricular pressure: $P_{\mathrm{ao}}$ : pressure immediately downstream from the AV; $P_{\text {art }}$ : simulated arterial pressure; $Q$ : flow rate.

was applied in three different configurations (named A, B, C) characterized by three different heights of application of the stitches. The three heights were identified by the surgeon on the sole basis of anatomical considerations, simulating a normal OR procedure:

- configuration A: the stitches were applied approximately at half the ITH (Fig. 1). This is the standard operating mode.

- configuration B: the stitches were applied empirically at $60 \%$ of the ITH, thus simulating a more aggressive AITR (Fig. 1). This setting was meant to analyze the effects of a mild alteration in the stitches position, either intentionally made by the surgeon to perform a more incisive surgery, or due to possible inter- or intra-operator variability in performing the surgical act. Again, the surgeon placed the stiches on the sole basis of anatomical inspection.

- configuration C: the stitches were applied empirically at 75\% of the ITH (Fig. 1). This was not intended to mimic a real clinical scenario, but was conceived as a limit case to extend the hemodynamic characterization even beyond the threshold usually considered in real AITR procedures.

\subsection{Experimental protocol}

Six fresh porcine AV samples were harvested by surgeons from porcine hearts. A 25-mm tract of the ascending aorta was preserved for housing the AV in the mock-loop test section and coronary arteries were ligated to avoid fluid loss.

Once housed in the mock-loop test section, each sample underwent a preliminary test in untreated condition (UC), then AITR was sequentially applied in the three configurations $(A, B, C)$ and tested. When each new configuration was made up, the stitches of the previously tested configuration were removed. All tests were carried out at a 70 -bpm pulse rate, using saline solution at $37^{\circ} \mathrm{C}$ as a working fluid and adjusting the hydraulic afterload to simulate physiologic normotensive conditions. Experimental mean flow rate was $4.4 \pm 0.4 \mathrm{~L} / \mathrm{min}$ and the measured mean simulated arterial pressure was $93 \pm 7 \mathrm{mmHg}$ (with diastolic pressure shifting from $93.1 \pm 8.1 \mathrm{mmHg}$ at the beginning of diastole to $84.1 \pm 7.6$ $\mathrm{mmHg}$ at the end of diastole). Pressure drop $(\Delta P)$ across the AV was evaluated as the difference between $P_{\text {ven }}$ and $P_{\text {ao }}$ (Fig. 2). From the raw hydrodynamic experimental data, the following quantities were evaluated and compared in all the tested configurations:

- $\Delta P_{\text {mean }}:$ mean systolic pressure drop across the AV.

- $\Delta P_{\max }$ : maximum systolic pressure drop across the AV.

- EOA: effective orifice area, calculated as follows:

$$
\mathrm{EOA}=\frac{Q_{\mathrm{rms}}}{3.1 \sqrt{\Delta P_{\text {mean }}}}
$$

where $Q_{r m s}$ is the root mean square value of the aortic flow rate.

- Ed\% $\%_{\text {sys: }}$ systolic dissipated energy.

- Ed\% dia: diastolic dissipated energy.

- $\mathrm{RF}_{\%}$ : regurgitant fraction, calculated from the telediastolic valve leak (Vismara et al., 2010).

At the end of each experimental test, the annulus diameter was measured with a go-no go gage (B1000, St. Jude Medical inc, St Paul, MN). Then, the AV samples were cut open along a generatrix and the positions the stitches were identified 
with a microscope. The actual heights at which the stitches were applied were evaluated from the microscopy images with the open-source image processing platform ImageJ (National Institute of Health, Bethesda, Maryland, USA).

\subsection{Statistical analysis}

Data were evaluated and averaged over 5 pulse cycles. The KolmogorovSmirnov test was used to assess normality. Data are presented as mean \pm standard deviation. Statistical analysis was performed with a one-way ANOVA test for repeated measures. A post-hoc Tukey test was performed to evaluate differences between groups and $P$-values $<0.05$ were considered statistically significant.

\section{Results}

Table 1 shows the anatomical measures and the post-surgery analysis of the actual stitches height. The expertise of the surgeon led to an excellent repeatability of the stitches placement, both among the three ITs and among the samples. Indeed, AITR was applied at $45 \pm 2 \% 61 \pm 4 \%$ and $79 \pm 6 \%$ of the ITH in configura-tions $A, B$ and $C$, respectively.

Hydrodynamic results are reported in Table 2, and Fig. 3 shows the trend of the main systolic indexes $\left(\Delta P_{\text {mean }}, \Delta P_{\text {max }}, E O A\right)$ as a function of the stitches position. When performed in configuration $A$, AITR didn't induce any statistically significant alteration of the hemodynamic performance of the AV with respect to the UC, with the exception of the EOA, which decreased from $3.6 \pm 1.4$ to $2.3 \pm 0.7$ $\mathrm{cm}^{2}(P<0.05)$. If compared to the UC, configuration $\mathrm{B}$ induced a statistically significant increase in the $\Delta P_{\text {mean }}$ (from $4.4 \pm 3.1$ to $13.5 \pm 6.9 \mathrm{mmHg}, P<0.05)$ and a reduction in the $\mathrm{EOA}(P<0.05)$, but no

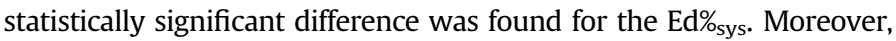
no statistically significant alteration was found between configurations $A$ and $B$ for any index.

On the other hand, configuration $C$ significantly altered the performance of the $A V$ with respect to each and every other condition. Indeed, the $\Delta P_{\text {mean }}$ and the $\Delta P_{\text {max }}$ increased up to
$29.2 \pm 7.8 \mathrm{mmHg}$ and $62.2 \pm 18.5 \mathrm{mmHg}$ respectively, and the Ed\% $\%_{\text {sys }}$ was $30.8 \pm 12.5 \%$.

As expected, AITR didn't influence the diastolic behavior of the $\mathrm{AV}$, i.e. its competence, in any of the experimental conditions, since both RF and Ed\% ${ }_{\text {dia }}$ did not change significantly among different groups.

\section{Discussion}

The reparative approach to AV pathologies has produced promising clinical evidence that valve repair is a suitable alternative to valve substitution (Aicher et al., 2010; Boodhwani et al., 2009a, 2009b). Nevertheless, if compared to prosthetic devices, surgical reparative procedures intrinsically lack standardization, owing to the absence of a structured and methodologically robust validation process to regulate their introduction in the clinical use. In particular, all commercial devices have to undergo a normalized approval process before clinical use, which usually involves a combination of in silico, in vitro and in vivo validation activities. Conversely, the evaluation and decision-making process for the standardization of a reparative technique is delegated to the surgeon, whose choice is generally based on anatomical/clinical considerations, and on experience.

The history of the introduction of AITR into the clinical practice well exemplifies this scheme. Cabrol was the first to describe this procedure in 1966 (Cabrol et al., 1966), yet without specific indications about the precise anatomical position at which the stitches should be placed. Lately, the AITR technique became widely adopted and other research groups further analyzed it from both the anatomical and functional points of view. It was recognized that AITR may have an important role for the long-term stabilization of AV repair, but the potential risk of inducing stenosis was also highlighted (El Khoury et al., 2005; Fraser and Cosgrove, 1994). However, no one up to now systematically

Table 1

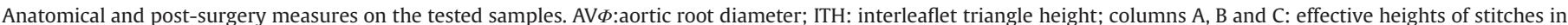

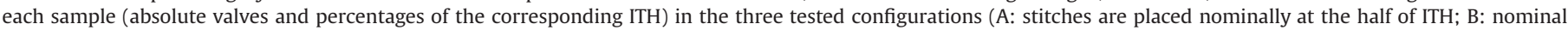
height of stiches 60\%; C: nominal height $80 \%$ ). Data are reported as mean \pm SD evaluated over the three ITs in each sample.

\begin{tabular}{|c|c|c|c|c|c|c|c|c|}
\hline & \multirow[t]{2}{*}{$\mathrm{AV} \phi[\mathrm{mm}]$} & \multirow[t]{2}{*}{ ITH [mm] } & \multicolumn{2}{|l|}{ A } & \multicolumn{2}{|l|}{ B } & \multicolumn{2}{|l|}{ C } \\
\hline & & & {$[\mathrm{mm}]$} & $\%$ & {$[\mathrm{~mm}]$} & $\%$ & {$[\mathrm{~mm}]$} & $\%$ \\
\hline AV \#1 & 21 & $15.2 \pm 0.2$ & $6.9 \pm 0.2$ & $45.5 \pm 1.1$ & $8.8 \pm 0.3$ & $58.0 \pm 1.2$ & $11.8 \pm 0.7$ & $78.1 \pm 5.4$ \\
\hline AV \#2 & 21 & $15.1 \pm 0.4$ & $6.9 \pm 0.2$ & $45.6 \pm 2.1$ & $8.4 \pm 0.3$ & $55.6 \pm 3.0$ & $10.7 \pm 0.6$ & $70.9 \pm 2.9$ \\
\hline AV \#3 & 23 & $15.3 \pm 0.6$ & $6.7 \pm 0.6$ & $43.5 \pm 3.3$ & $9.8 \pm 1.3$ & $64.0 \pm 6.5$ & $13.0 \pm 1.3$ & $84.7 \pm 5.4$ \\
\hline AV \#4 & 27 & $18.2 \pm 0.3$ & $8.0 \pm 0.0$ & $44.0 \pm 0.7$ & $11.2 \pm 0.3$ & $61.5 \pm 2.2$ & $14.2 \pm 0.8$ & $78.0 \pm 3$ \\
\hline AV \#5 & 19 & $13.3 \pm 1.5$ & $6.2 \pm 1.3$ & $45.9 \pm 4.2$ & $8.3 \pm 0.6$ & $62.7 \pm 3.5$ & $10.7 \pm 0.6$ & $80.4 \pm 6.2$ \\
\hline \multirow[t]{2}{*}{ AV \#6 } & 19 & $17.3 \pm 1.2$ & $7.8 \pm 0.8$ & $45.1 \pm 1.8$ & $10.7 \pm 0.8$ & $61.6 \pm 2.9$ & $14.5 \pm 0.9$ & $83.7 \pm 0.6$ \\
\hline & $22 \pm 3$ & $15.7 \pm 1.8$ & $7.2 \pm 0.7$ & $45.0 \pm 2.1$ & $9.7 \pm 1.2$ & $60.6 \pm 4.0$ & $12.9 \pm 1.9$ & $79.3 \pm 6.1$ \\
\hline
\end{tabular}

Table 2

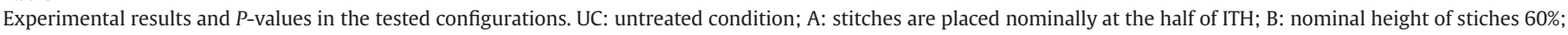

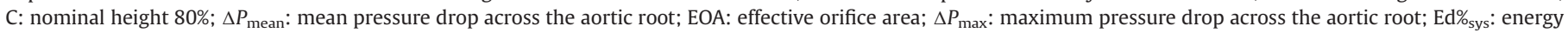
dissipated in the systole; RF\%: regurgitant fraction, calculated from the telediastolic valve leakage; Ed\% ${ }_{\text {dia }}$ : energy dissipated in the diastole.

\begin{tabular}{|c|c|c|c|c|c|c|c|c|c|c|}
\hline & \multirow[t]{2}{*}{ UC } & \multirow[t]{2}{*}{ A } & \multirow[t]{2}{*}{ B } & \multirow[t]{2}{*}{ C } & \multicolumn{6}{|l|}{$P$-values } \\
\hline & & & & & UC vs A & UC vs B & UC vs C & A vs B & A vs C & B vs C \\
\hline$\Delta \boldsymbol{P}_{\text {mean }}[\mathrm{mmHg}]$ & $4.4 \pm 3.1$ & $9.3 \pm 5.4$ & $13.5 \pm 6.9$ & $29.2 \pm 7.8$ & $n s$ & $P<0.05$ & $P<0.05$ & $n s$ & $P<0.05$ & $P<0.05$ \\
\hline$E O A\left[\mathrm{~cm}^{2}\right]$ & $3.6 \pm 1.4$ & $2.3 \pm 0.7$ & $1.9 \pm 0.6$ & $1.2 \pm 0.1$ & $P<0.05$ & $P<0.05$ & $P<0.05$ & $n s$ & $n s$ & $n s$ \\
\hline$\Delta P_{\max }[\mathrm{mmHg}]$ & $25.3 \pm 6.3$ & $31.7 \pm 9.0$ & $35.3 \pm 8.5$ & $62.2 \pm 18.5$ & $n s$ & $n s$ & $P<0.05$ & $n s$ & $P<0.05$ & $P<0.05$ \\
\hline Ed\% $\%_{\text {sys }}$ & $5.3 \pm 3.3$ & $9.8 \pm 5.1$ & $13.8 \pm 5.8$ & $30.8 \pm 12.5$ & $n s$ & ns & $P<0.05$ & $n s$ & $P<0.05$ & $P<0.05$ \\
\hline $\mathbf{R F}_{\%}$ & $4.1 \pm 2.3$ & $5.1 \pm 2.7$ & $5.2 \pm 2.3$ & $7.1 \pm 3.0$ & $n s$ & ns & ns & ns & $n s$ & $n s$ \\
\hline Ed\% $\%_{\text {dia }}$ & $5.4 \pm 2.0$ & $5.8 \pm 1.9$ & $5.3 \pm 1.9$ & $5.4 \pm 1.9$ & $n s$ & $n s$ & $n s$ & $n s$ & $n s$ & $n s$ \\
\hline
\end{tabular}



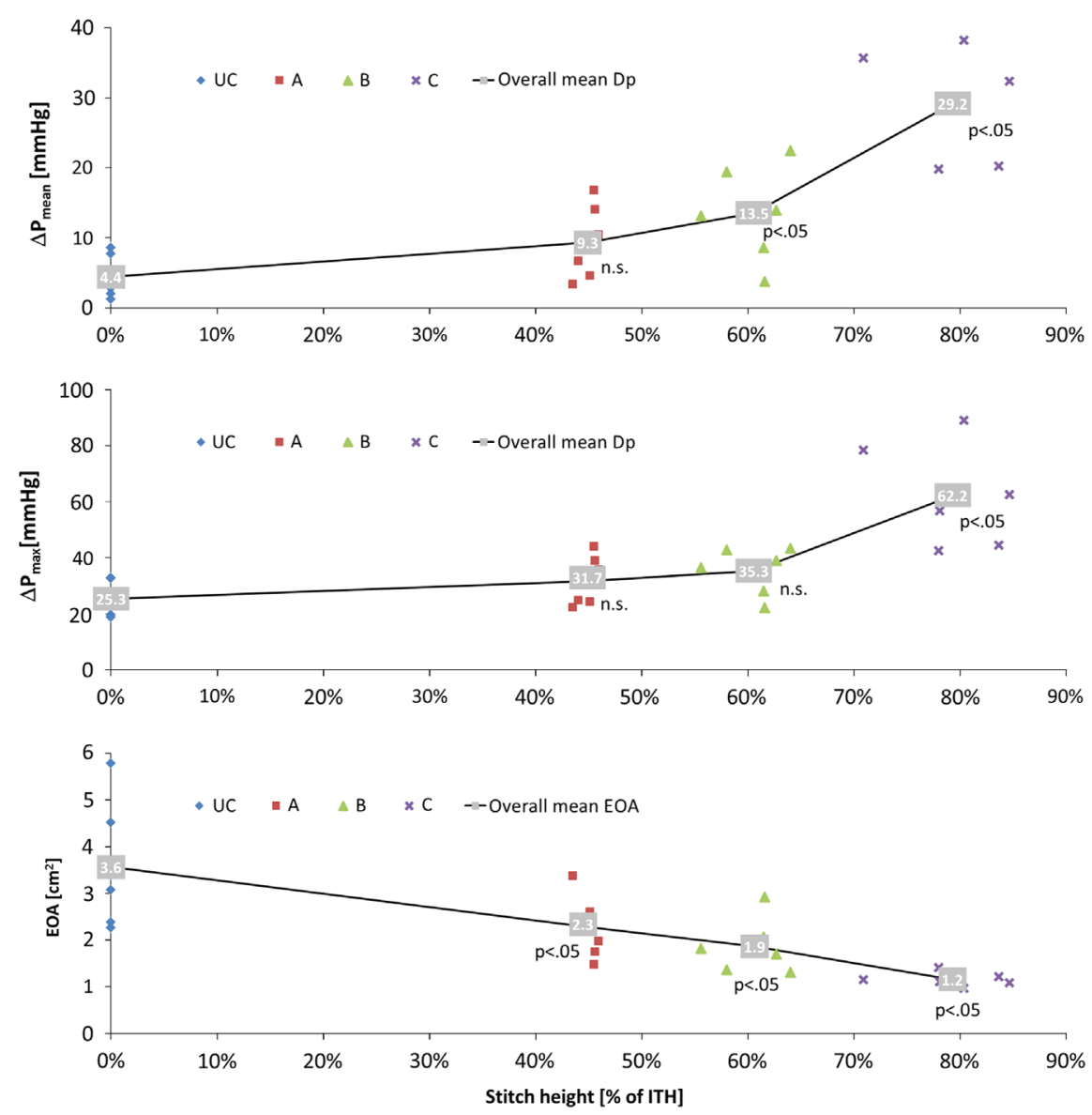

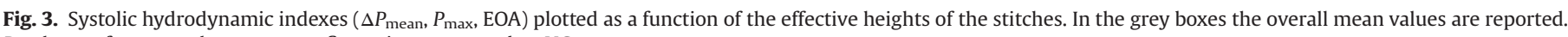
$P$-values refers to each surgery configuration compared to UC.

investigated the effects of changing the position of the stitches to find an optimum, such optimum being a trade-off between the goal of achieving stabilization (by increasing the coaptation area reserve) and the need to keep an adequate AV orifice.

In a previous computational study, it was shown that AITR performed at $48 \%$ of the ITH is optimal in terms of restoration of the physiologic coaptation area. In this work, we adopted an in vitro approach to investigate the effects of AITR stitch positioning on AV hemodynamic behaviour. In particular, we wanted to quantify the relation existing between the stitch positioning height and the systolic performance of the AV in order to estimate the safety margin that the surgeon can rely on in the operating room.

Our results showed that AITR performed at about $45 \%$ of the ITH only induces a mild reduction of EOA, without causing statistically significant alteration in any other hemodynamic index. This finding is in agreement with a previous in vitro study that compared different treatments for AV insufficiency (De Kerchove et al., (2012)). When performed in a more central configuration, i.e. at $60 \%$ of the ITH, AITR also induced a minor increase in the mean transvalvular pressure drop. Finally, as expected, when performed at $80 \%$ of the ITH, AITR induced a severe AV stenosis.

In general, all findings were in agreement with the inferences that may be drawn from the analysis of the structural shape of the ITs. The trends of the systolic hemodynamic indexes (Fig. 3) ideally replicate the non-linear anatomical divergence of the IT sides (Fig. 2), thus confirming that a direct correlation exists between the hemo-dynamic performance of the AV and the entity of the annuloplasty.

These in vitro results have interesting implications for the clinical application of AITR. On the one hand, indeed, the hemodynamic findings related to configuration $\mathrm{A}$, together with our previous results (De Kerchove et al., (2012); Mangini et al., 2011), suggest that the optimal height for the surgery is around $45-50 \%$ of the ITH. Moreover, it appears that placing the stitches at this optimal level implies a limited risk of inducing AV stenosis in the post-op scenario, or, that the surgeon can rely on a reasonable safety margin when executing the procedure in the OR.

Even if it wasn't the aim of this study to evaluate the precision of the surgical act, our measurements yielded some indications about the possible variability of stitch positioning. For the conservative configuration (A), there was a systematic tendency of the surgeon to underestimate the half-ITH position (45\% rather than $50 \%$ ). Still the repeatability of stitch positioning was fairly high, with a standard deviation of the actual stitch height among the three leaflets (Table 1 ) always lower than $5 \%$ of the ITH. Considering that even in configuration B the induced stenosis is still moderate, and that no statistically significant difference was found between configurations $\mathrm{A}$ and $\mathrm{B}$, one can infer that configuration $\mathrm{A}$ is reasonably safe, even taking into account the variability associated with the surgical act.

Conversely, the non-linear trends of the systolic indexes seem to suggest that a threshold exists between configurations $\mathrm{B}$ and $\mathrm{C}$, beyond which small differences in the stitches position may induce relevant alterations on the hydrodynamic behavior. There-fore, the surgeon performing a more central AITR should be aware that the safety margin he can rely on is reduced. This is due to the diverging anatomy of the cusps insertion lines and is confirmed by the significant change in the indexes that was measured with AITR performed at $80 \%$.

The main limitation of the present study is that the in vitro campaign was carried out on isolated healthy porcine AV samples, i.e. without the use of a pathological model. 
Nonetheless, the rationale of our approach was to consider the healthy AV as a model of an optimally treated pathological valve, i.e. a valve whose functional anatomy has been perfectly restored by a state-of-the-art reparative procedure (Vismara et al., 2011). Indeed AITR is typically intended as a technique for the long-term stabilization of the short-term results of traditional reparative techniques, rather than a repair technique per se. Hence, it is typically applied after conventional surgical repair of the ARFU, when correct AV competence is already restored. In this vision, AITR is intended to prevent further AVJ dilatation, thus allowing correct leaflet coaptation and avoiding the risk of recurrent AV insufficiency.

Using healthy AV samples in our tests, instead of surgically corrected pathological valves, was the result of a design tradeoff, where the need for replicating in vitro the clinical complexity conflicted with the pursuit for a total control of the experimental settings. This approach somewhat affected the possibility to directly transfer our results to all the scenarios the surgeon can face in the OR, but it allowed us to isolate the effects of AITR on valve function in a very selective manner, giving our data the powerfulness of repeatability. Theoretically, considering AITR as an ancillary technique to stabilize post-op results, then there should be no reasons to omit it, unless it provably alters the valve functioning in a clinically relevant manner. Our study suggests that the AITR, performed at half of the ITH, does not alter the valve functioning significantly per se, when applied to a valve whose repaired anatomy is comparable to the physiologic one. To date, this conclusion had been mainly based on surgeons' experience and knowledge of AV functional anatomy. Our study provides this indication within a simplified scenario, but, for the first time to our knowledge, with a reliable and sound set of in vitro experimental data, providing also indication of the safety margin the surgeon can rely on.

\section{Conflict of interest statement}

The authors have no conflict of interest to disclose.

\section{References}

Aicher, D., Fries, R., Rodionycheva, S., Schmidt, K., Langer, F., Schafers, H.J., 2010. Aortic valve repair leads to a low incidence of valve-related complications. Eur.J. Cardiothorac. Surg. 37, 127-132.
Boodhwani, M, de Kerchove, L., Glineur, D., Poncelet, A., Rubay, J., Astarci, P., Verhelst, R., Noirhomme, P., El Khoury, G., 2009a. Repair-oriented classification of aortic insufficiency: impact on surgical techniques and clinical outcomes.

J. Thorac. Cardiovasc. Surg. 137, 286-294.

Boodhwani, Munir, de Kerchove, L., Glineur, D., Poncelet, A., Rubay, J., Astarci, P., Verhelst, R., Noirhomme, P., El Khoury, G., 2009b. Repair-oriented classification of aortic insufficiency: impact on surgical techniques and clinical outcomes. J. Thorac. Cardiovasc. Surg. 137, 286-294.

Cabrol, C., Cabrol, A., Guiraudon, G., Bertrand, M., 1966. Treatment of aortic insufficiency by means of aortic annuloplasty. Arch. Mal. Coeur. Vaiss. 59, 13051312.

Cheng, A., Dagum, P., Miller, D.C., 2007. Aortic root dynamics and surgery: from craft to science. Philos. Trans. R. Soc. London, Ser. B 362, 1407-1419.

De Kerchove, L., Vismara, R., Mangini, A., Fiore, G.B., Price, J., Noirhomme, P., Antona, C.,

El Khoury, G., 2012. In vitro comparison of three techniques for ventriculoaortic junction annuloplasty. Eur. J. Cardiothorac. Surg. 41, 1117-1124

El Khoury, G., Glineur, D., Rubay, J., Verhelst, R., D'Acoz, Y., d'Udekem, Poncelet, A., Astarci, P., Noirhomme, P., van Dyck, M., 2005. Functional classification of aortic root/valve abnormalities and their correlation with etiologies and surgical procedures. Curr. Opin. Cardiol. 20, 115-121.

Fraser Jr., C.D., Cosgrove 3rd, D.M., 1994. Surgical techniques for aortic valvuloplasty. Tex. Heart Inst. J. 21, 305-309.

Hopkins, R.A., 2003. Aortic valve leaflet sparing and salvage surgery: evolution of techniques for aortic root reconstruction. Eur. J. Cardiothorac. Surg. 24, 886-897.

Langer, F., Aicher, D., Kissinger, A., Wendler, O., Lausberg, H., Fries, R., Schäfers, H.-J. 2004. Aortic valve repair using a differentiated surgical strategy. Circulation 110, II67-II73.

Lanzarone, E., Vismara, R., Fiore, G.B., 2009. A new pulsatile volumetric device with biomorphic valves for the in vitro study of the cardiovascular system. Artif. Organs 33, 1048-1062.

Mangini, A., Lemma, M.G., Soncini, M., Votta, E., Contino, M., Vismara, R., Redaelli,

A., Antona, C., 2011. The aortic interleaflet triangles annuloplasty: a multidisciplinary appraisal. Eur. J. Cardiothorac. Surg. 40, 851-857.

Mve Mvondo, C., Nardi, P., Bassano, C., Bertoldo, F., Grego, S., D'Auria, F., Scafuri, A., Chiariello, L., 2013. Surgical treatment of aortic valve regurgitation secondary to ascending aorta aneurysm: is adjunctive subcommissural annuloplasty necessary? Ann. Thorac. Surg. 95, 586-592.

Vahanian, A., Baumgartner, H., Bax, J., Butchart, E., Dion, R., Filippatos, G., Flachskampf, F., Hall, R., Iung, B., Kasprzak, J., Nataf, P., Tornos, P., Torracca, L., Wenink, A., 2007. Guidelines on the management of valvular heart disease: the task force on the management of valvular deart disease of the European Society of Cardiology. Eur. Heart J. 28, 230-268.

Vismara, R., Antona, C., Mangini, A., Cervo, M., Contino, M., Bosisio, E., Redaelli, A., Fiore, G.B., 2011. In vitro study of aortic valves treated with neo-chordae grafts: hydrodynamics and tensile force measurements. Ann. Biomed. Eng. 39, 1024-1031.

Vismara, R., Fiore, G.B., Mangini, A., Contino, M., Lemma, M., Redaelli, A., Antona, C., 2010. A novel approach to the in vitro hydrodynamic study of the aortic valve: mock loop development and test. ASAIO J. 56, 279-284.

Vismara, R., Mangini A., Romagnoni C., Contino M., Redaelli A., Fiore G.B., A.C., In vitro study of a porcine quadricuspid aortic valve. J. Heart Valve Dis. In press. Yacoub, M.H., Kilner, P.J., Birks, E.J., Misfeld, M., 1999. The aortic outflow and root: a tale of dynamism and crosstalk. Ann. Thorac. Surg. 68, S37-S43. 